\title{
Pseudomicetoma dermatofítico en una gata persa: reporte de un caso en Costa Rica
}

\section{Dermatophytic pseudomycetoma in a Persian cat: case report in Costa Rica}

\section{Pseudomicetoma dermatofítico em uma gata persa: relato de um caso em Costa Rica}

\author{
Natalia Sandoval Salazar ${ }^{1}$, Alejandra Calderón-Hernández ${ }^{1 凶}$, Karla Mora Alfaro ${ }^{2}$, Stephanie Unger ${ }^{3}$,
} Juan A. Morales ${ }^{4}$

1 Laboratorio de Micología, Escuela de Medicina Veterinaria, Universidad Nacional, Heredia, Costa Rica. natisand25@gmail.com, alejandra.calderon.hernandez@una.cr

2 Centro Veterinario Dr. Jimmy, City Mall Alajuela, Costa Rica. kmoralafaro@gmail.com

3 Ecovet, Ecografía Veterinaria, San José, Costa Rica. ecodraunger@gmail.com

4 Servicio de Patología, Escuela de Medicina Veterinaria, Universidad Nacional, Heredia, Costa Rica. juan.alberto.morales.emv@gmail.com

Recibido: 16 de enero de 2020 Corregido: 20 de abril de 2020 Aceptado: 01 de mayo de 2020

\begin{abstract}
Resumen
Se describe un caso de pseudomicetoma dermatofítico por Microsporum canis demostrado mediante histopatología con H\&E, GMS y PAS, examen directo con $\mathrm{KOH}$ y cultivo micológico en un felino, persa, hembra, castrada, de 5 años, con historia de múltiples masas subcutáneas, indoloras y recidivantes en el dorso durante año y medio. Se trató con itraconazol durante cuatro meses, pero se suspendió por intolerancia al medicamento. La invasión a órganos internos se descartó mediante ultrasonido y citología.

Palabras clave: felino, gato persa, itraconazol, Microsporum canis, pseudomicetoma.

Abstract

The paper describes a case of dermatophytic pseudomycetoma caused by Microsporum canis diagnosed through histopathology with H\&E, GMS, and PAS, direct microscopic examination with $\mathrm{KOH}$, and mycological culture in a spayed 5-year-old female Persian feline, with a history of multiple recurrent nontender subcutaneous masses on the back over a year and a half. The subject was treated with Itraconazole for four months, which was suspended due to drug intolerance. Internal organ invasion was discarded by ultrasound and cytology.
\end{abstract}

Keywords: feline, Persian cat, itraconazole, Microsporum canis, pseudomycetoma.

\ Correo de correspondencia: $\underline{\text { alejandra.calderon.hernandez@una.cr }}$ 


\begin{abstract}
Resumo
O artigo descreve um caso de pseudomicetoma dermatofítico causado por Microsporum canis diagnosticado através de histopatologia com H\&E, GMS e PAS, exame direto com $\mathrm{KOH}$ e cultura micológica em um felino, persa, fêmea, castrado, de 5 anos de idade, com histórico de múltiplas massas subcutâneas, indolor e recorrentes localizadas no dorso do animal, por mais de um ano e meio. O paciente foi tratado com Itraconazol por quatro meses, o qual foi suspenso devido à intolerância ao medicamento. A invasão de órgãos internos foi descartada por ultrassom e citologia.
\end{abstract}

Palavras-chave: felino, gato persa, itraconazol, Microsporum canis, pseudomicetoma.

\title{
Introducción
}

El pseudomicetoma dermatofítico es una infección micótica cutánea profunda o subcutánea, poco frecuente, cuyo principal agente causal es Microsporum canis (Chang et al. 2010; Bianchi et al. 2017; Duangkaew et al. 2017). Esta micosis se manifiesta, clínicamente, en forma de nódulos dérmicos, generalmente ulcerados, de ocurrencia única o múltiple, de diversos tamaños; pueden presentar una secreción granular y aparecen sin historia previa de traumatismo cutáneo (Chang et al. 2010; Nobre et al. 2010).

Se ha descrito, esta micosis, en varias especies; entre ellas: caninos, felinos, equinos e incluso humanos (Bond et al. 2001; Pereira et al. 2006). Sin embargo, ha sido reportada con mayor frecuencia en gatos persa, los cuales, se presume, están predispuestos a esta condición debido a una deficiencia inmune hereditaria, al microambiente cutáneo que poseen o a un acicalado ineficiente debido a lo largo del pelaje (Stanley et al. 2008; Duangkaew et al. 2017).

En felinos, las lesiones, usualmente, se localizan en: la piel del tórax dorsal y ventral, cabeza, cuello, abdomen, cola y miembros anteriores y posteriores; también, existen reportes de pseudomicetomas en la región sublumbar, intra-abdominal y en linfonodos (Bianchi et al. 2017; Sousa Filho et al. 2017).

Entre los principales diagnósticos diferenciales se puede citar etiologías infecciosas y no infecciosas que causan nódulos en piel: feohifomicosis, criptococosis, esporotricosis, histoplasmosis, nocardiosis, actinomicosis, micobacterias atípicas, neoplasias, abscesos y reacciones post-vacunales (Pereira et al. 2006; Sousa Filho et al. 2017).

Microsporum canis es un hongo que presenta distribución mundial; por lo cual, existen reportes de caso de pseudomicetomas en países tan variados como: Brasil, Taiwán, Tailandia e Inglaterra (Bond et al. 2001; Chang et al.2010; Bianchi et al. 2017; Duangkaew et al. 2017). En Costa Rica, esta patología podría presentarse, de forma esporádica, debido a que este hongo es el principal causante de dermatofitosis en perros y gatos (Calderón-Hernández \& Urbina-Villalobos 2018); pero, quizá por desconocimiento de la enfermedad no está siendo diagnosticada. Por tal razón, se describe el siguiente caso clínico, con el propósito de que los médicos veterinarios, de pequeñas especies, de Costa Rica y Centroamérica consideren el pseudomicetoma en el diagnóstico diferencial de masas subcutáneas en felinos, principalmente los de raza persa.

\section{Reporte de Caso}

El 18 de enero de 2019, un felino de raza persa, de 5 años, hembra, castrada, de $3.3 \mathrm{Kg}$ de peso, se llevó a consulta al Centro Veterinario Dr. Jimmy, en City Mall, Alajuela, Costa Rica, presentando varias masas granulomatosas en el dorso, con historia de recidiva, después de la remoción quirúrgica. 
La felina fue castrada cuando era cachorra, no sale de casa, la última vez que se había escapado fue aproximadamente dos años y había regresado con arañazos. El paciente, convive con dos gatos himalaya de cinco meses y seis años, respectivamente; que no presentaban lesiones. Los propietarios no tenían historia de lesiones en piel tampoco. A la consulta, el animal presentaba seis masas de aspecto granulomatoso, una coalescente en la región lumbar, dorsal a la base de la cola, de aproximadamente $7 \times 6 \mathrm{~cm}$; otra en la escápula izquierda de $5 \times 4 \mathrm{~cm}$; otra en la escápula derecha de $4 \times 4 \mathrm{~cm}$ y dos más caudales al pabellón auricular derecho de $0.5 \times 0.5 \mathrm{~cm}$ cada una. Todos los nódulos eran indoloros; tenían apariencia redondeada, no adheridos al tejido adyacente y suaves a la palpación (Fig. 1).
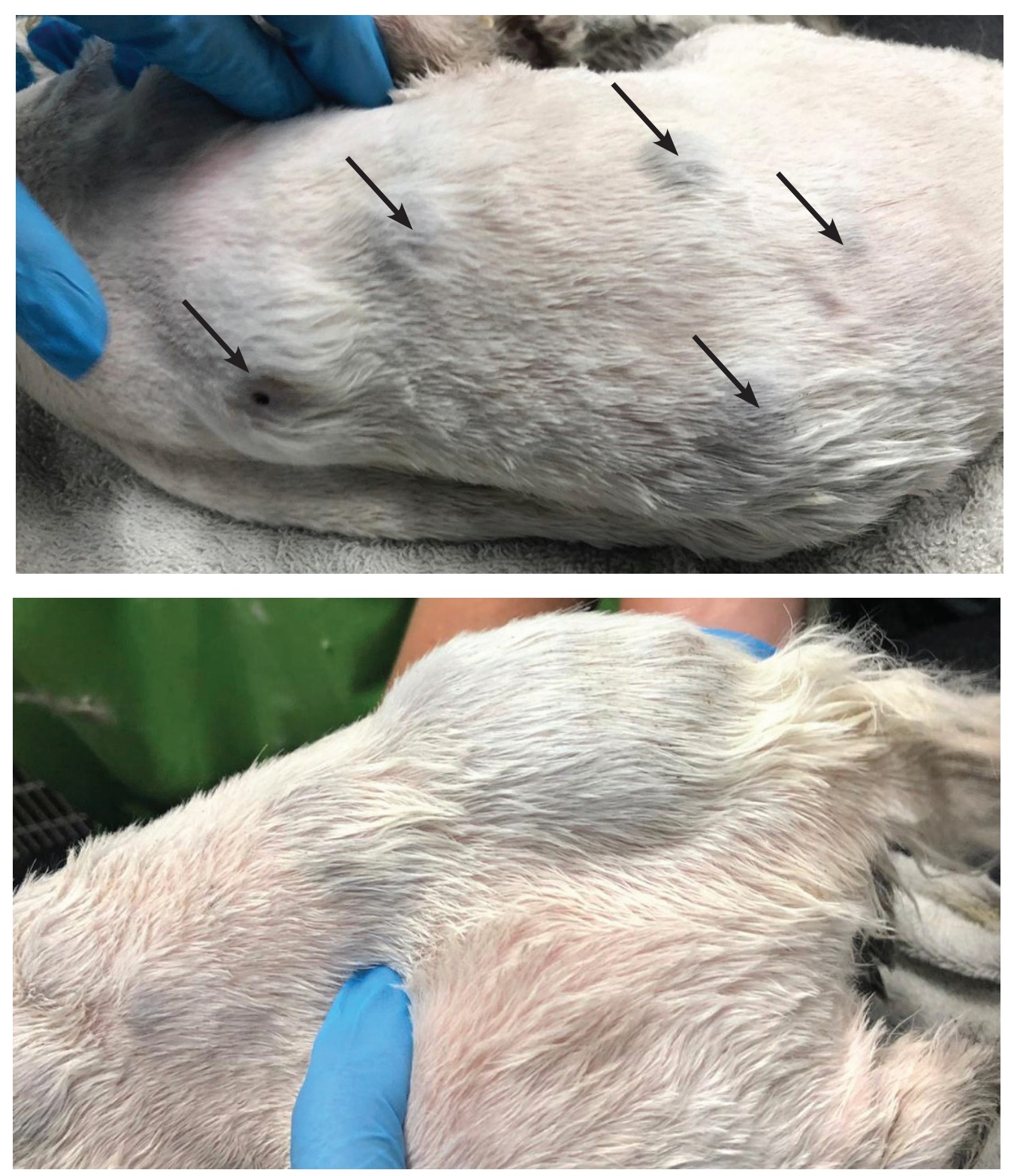

Fig. 1. A. Múltiples nódulos ubicados en el dorso del animal (flechas). B. Masa craneal a la cola (flecha). Cortesía Dra. Rebeca Orias. 


\section{Hematología, química clínica y serología}

Se realizó hemograma y químicas sanguíneas utilizando el VetScan ${ }^{\circledR}$ VS2 Chemistry Analyzer (Abaxis, California, Estados Unidos), en las cuales, las únicas alteraciones, en los análisis, fue una leve leucopenia que se asocia a estrés y un leve incremento en la Alanina Aminotransferasa (ALT) (241 U/L, rango 20100). Así mismo, se llevó a cabo una prueba serológica de Leucemia e Inmunodeficiencia felina (FIV/ FeLV Anigen ${ }^{\circledR}$ Veterinary Test, Bionote Inc., Seúl, Corea) la cual resultó negativa.

\section{Histopatología}

Se decide remover, quirúrgicamente, una masa de la región escapular derecha para histopatología, la cual fue fijada en formalina bufferada al 10\% y analizada en el Servicio de Patología de la Escuela de Medicina Veterinaria de la Universidad Nacional de Heredia, Costa Rica (EMV-UNA).

Mediante la tinción de Hematoxilina y Eosina (H\&E), a nivel de la epidermis, no se observó alteraciones; en la dermis superficial y profunda, se observaron múltiples áreas focales de necrosis y un proceso inflamatorio pio-granulomatoso delimitado por proliferación de tejido conectivo. El proceso inflamatorio se caracteriza por masiva infiltración de macrófagos y neutrófilos, en menor grado la presencia de células sincitiales (células gigantes). En la zona de necrosis e inflamación se observa la presencia de estructuras de tipo micótico, amorfas con dilataciones bulbosas (Fig. 2).

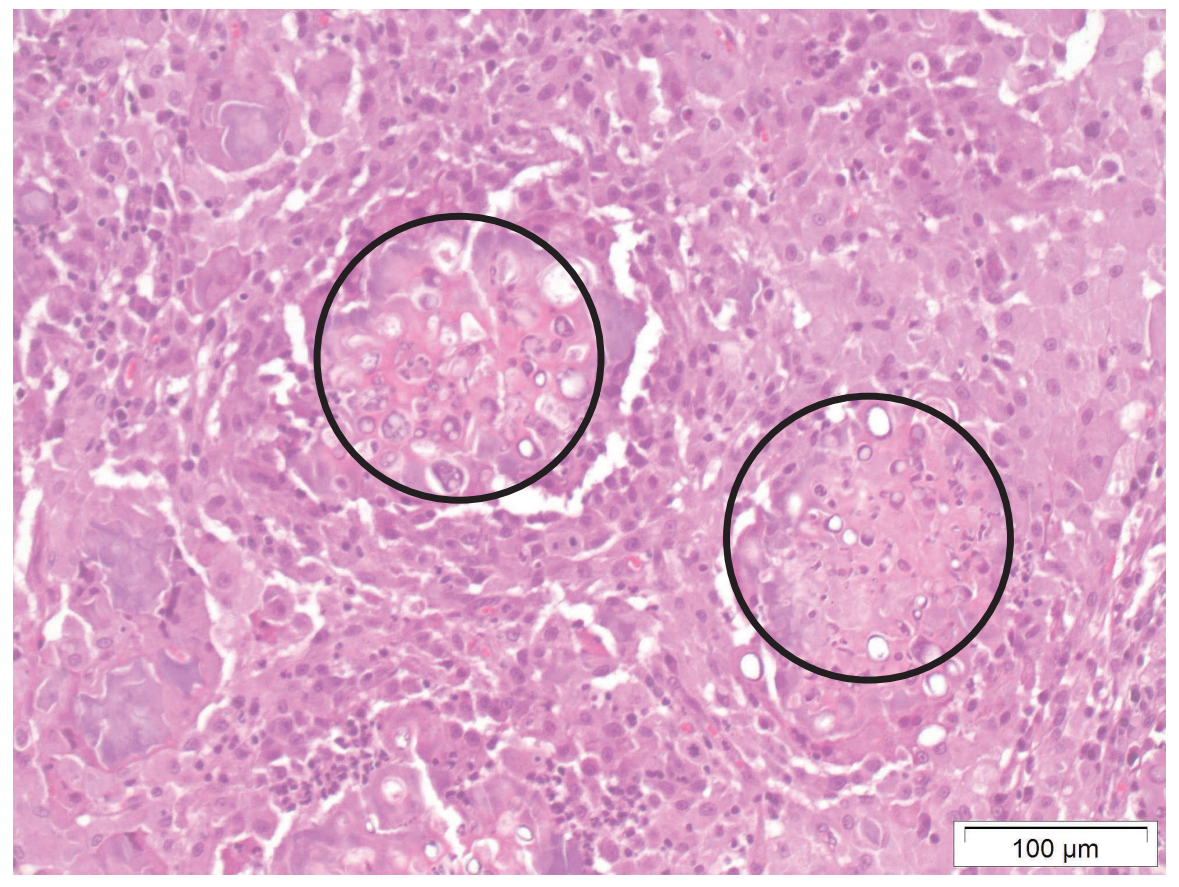

Fig. 2. Histopatología de una masa en piel donde delimitado por círculos, se observa áreas de necrosis rodeadas de polimorfonucleares, con presencia de estructuras micóticas bulbosas. H\&E, 200x 
Se realizó las tinciones de Ácido Peryódico de Schiff(PAS) y Tinción Plata Metenamina de Grocott (GMS), para diferenciar mejor las estructuras micóticas. En dichas preparaciones, se pudo observar que la pared de las estructuras fúngicas se tiñó positivamente de color fucsia en el caso del PAS y de color negro con el GMS (Walsh et al. 2018b), confirmando, así, la presencia de hifas y pseudohifas de hongos de paredes no paralelas, tamaños y grosores irregulares, en las cuales no se observó ramificación ni se logró distinguir si eran septadas (Fig. 3 y 4 ).

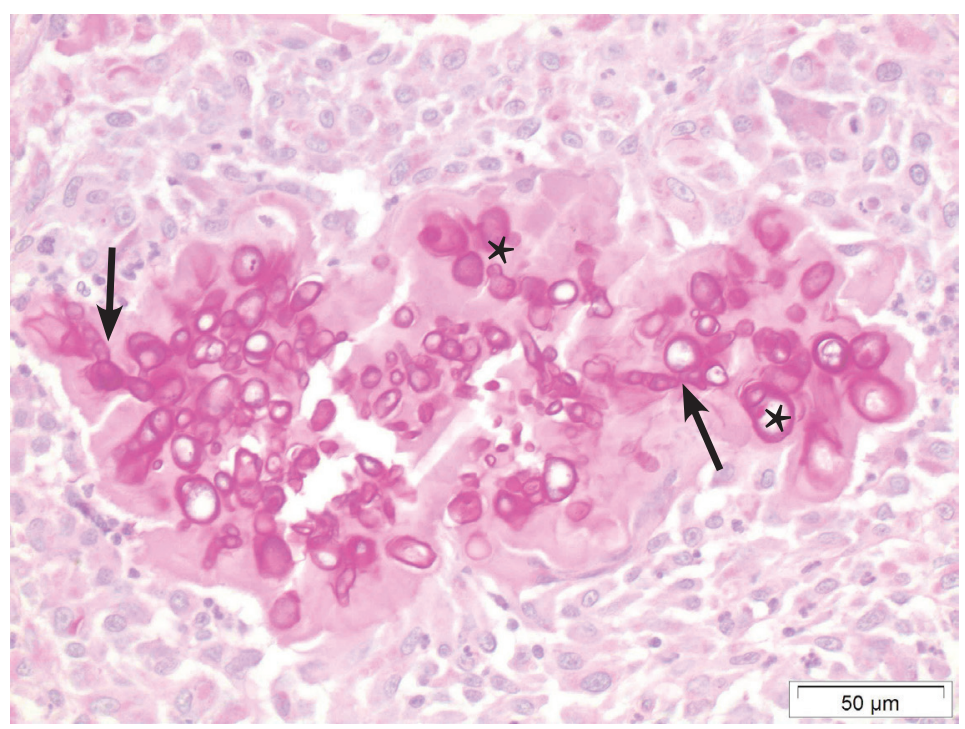

Fig. 3. Histopatología de un pseudomicetoma donde se observa la presencia de estructuras micóticas de bulbosas (asteriscos) y pseudohifas (flechas), delimitado por infiltración celular a base de macrófagos. PAS, 400x.

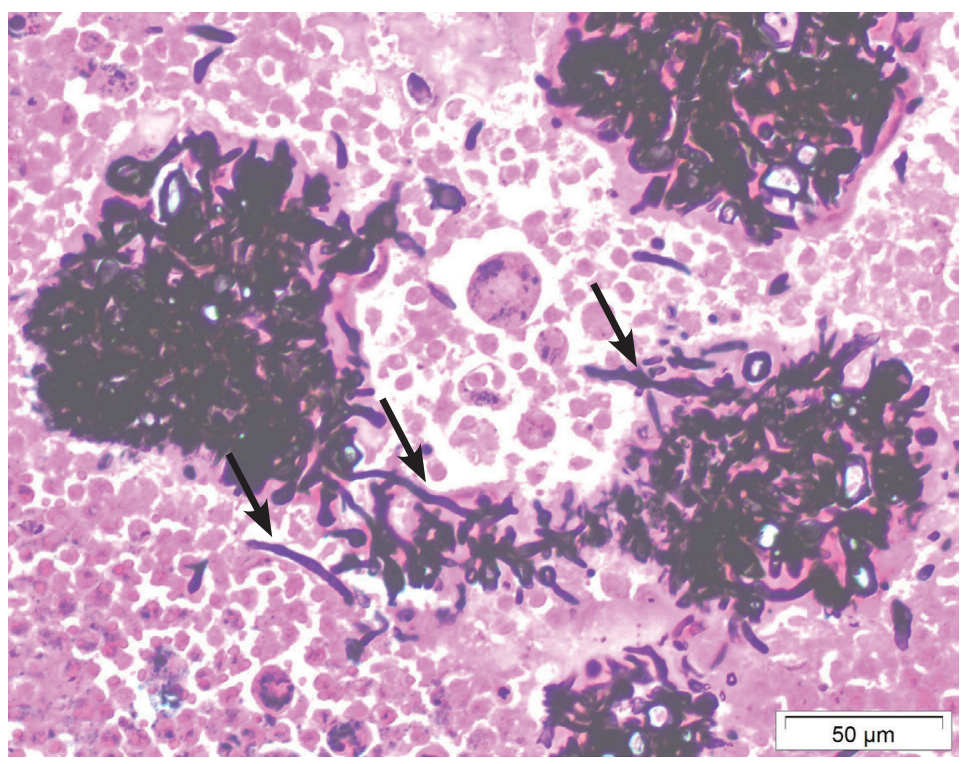

Fig. 4. Corte histopatológico de un pseudomicetoma en el cual se observa la presencia de hifas de hongos de varios grosores (flechas). GMS, 400x. 


\section{Análisis micológicos}

Una vez obtenidos los resultados de la biopsia, el 25 de enero de 2019, se toma una biopsia de una de las masas caudales al pabellón auricular (Fig. 5), muestra de barrido del pelambre mediante la técnica de Mackenzie, adaptada por Goldberg (1965) y uñas, tanto de este animal como de los otros dos gatos que conviven con ella, para determinar si eran portadores asintomáticos de dermatofitos. Los análisis micológicos se realizaron en el Laboratorio de Micología de la EMV-UNA.

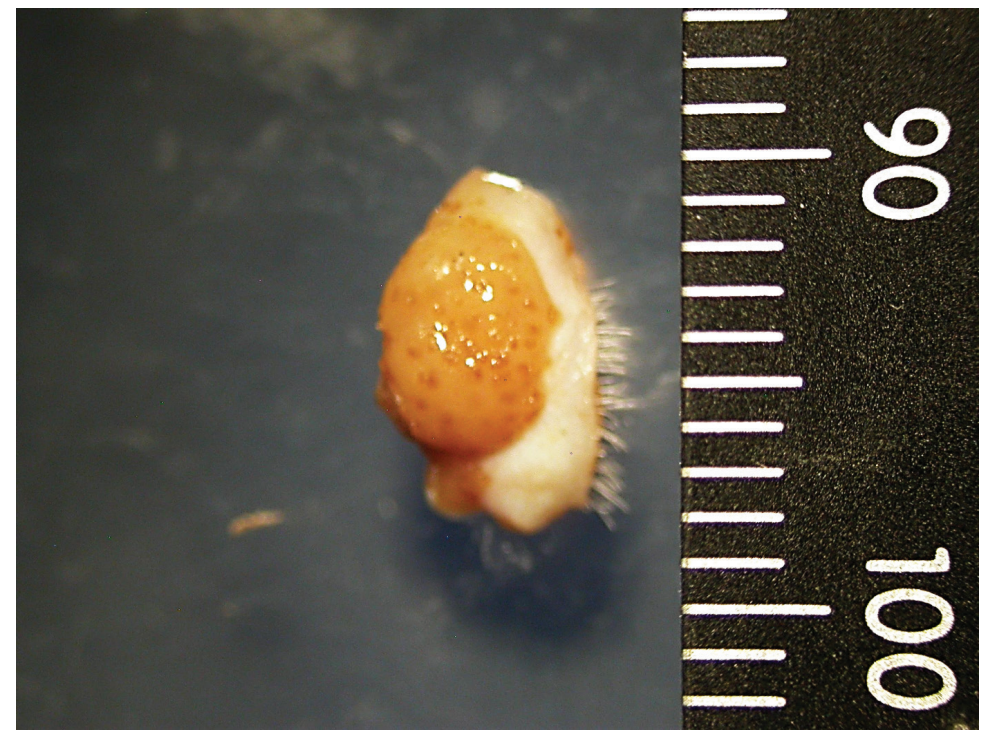

Fig. 5. Aspecto macroscópico de la biopsia remitida para análisis micológico. Nótese la presencia de múltiples nódulos en su interior.

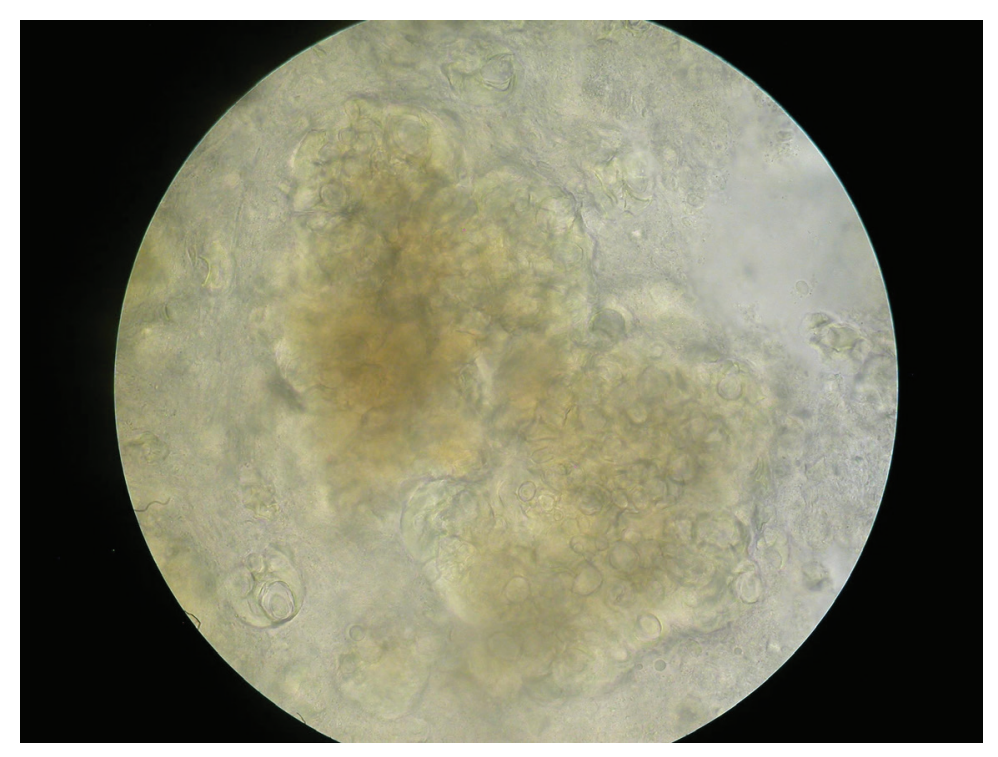

Fig. 6. Pseudomicetoma de hongos hialinos. Examen microscópico directo con $\mathrm{KOH} 40 \%$, 400X. 
A partir de la biopsia, se realizan dos cultivos: el primero en Agar Dermasel (Oxoid ${ }^{\circledR}$, Basingstoke, Reino Unido) a $28^{\circ} \mathrm{C}$; el segundo en Agar Sabouraud Dextrosa (Oxoid ${ }^{\circ}$, Basingstoke, Reino Unido) a $37^{\circ} \mathrm{C}$. Así mismo, se realiza un examen microscópico directo con hidróxido de potasio $(\mathrm{KOH})$ al $40 \%$, en el cual se observa abundantes pseudomicetomas de hongos hialinos (Fig. 6).

Las muestras de cepillado, y de las uñas, fueron cultivadas en Agar Dermasel a $28^{\circ} \mathrm{C}$. Todos los cultivos se incubaron durante 15 días y fueron revisados cada dos días.

Los cultivos de las muestras del cepillado y las uñas fueron negativos; mientras que, a los 14 días (8 de febrero de 2019), en el cultivo del tejido, se observó un crecimiento en ambos agares, de un hongo hialino algodonoso, de crecimiento radiado en el anverso y hialino con pigmento amarillo en el reverso (Fig. 7).
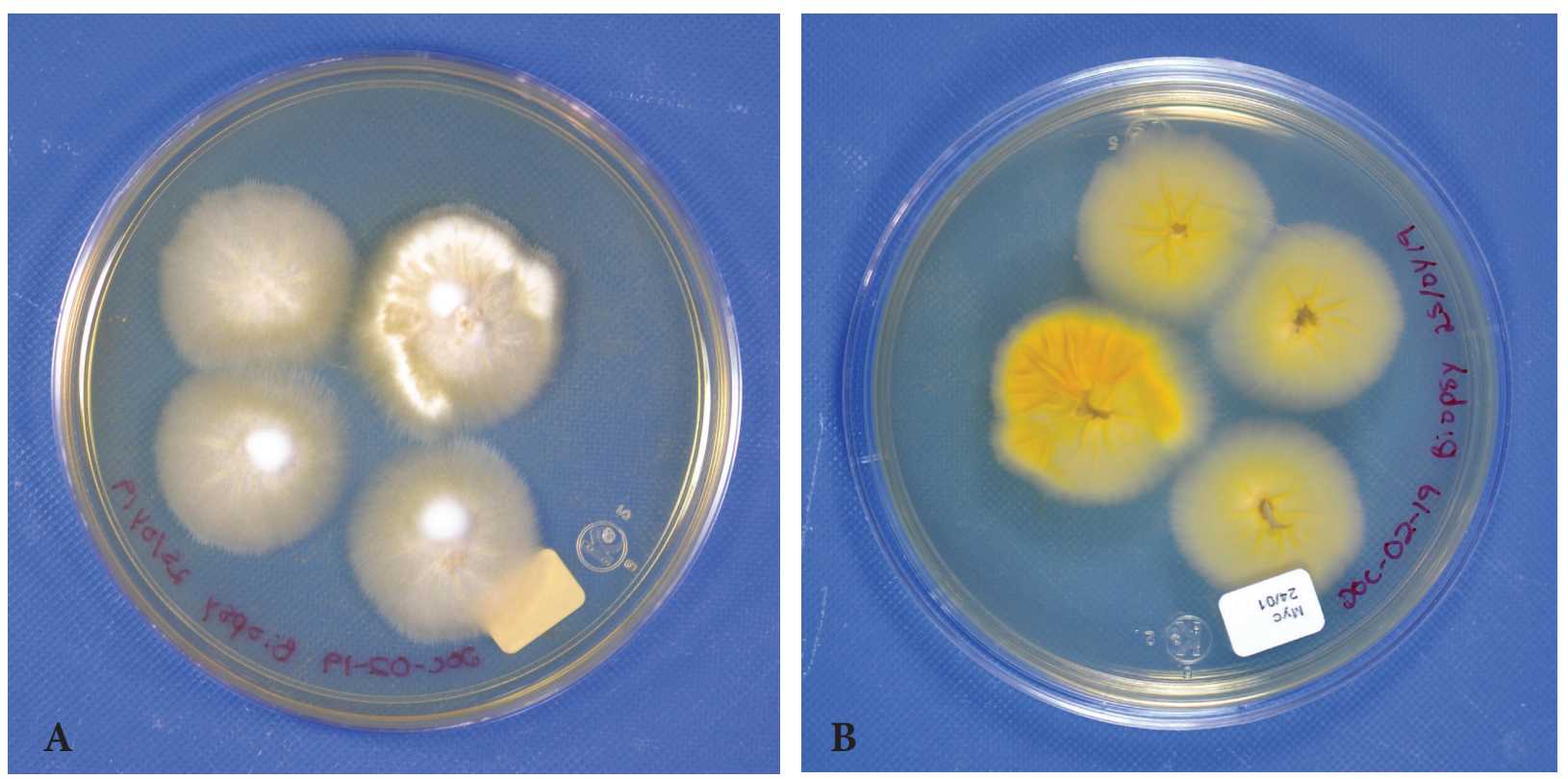

Fig. 7. Cultivo micológico de la muestra de biopsia en Agar Dermasel a $28{ }^{\circ} \mathrm{C}$ a los 14 días. A. Anverso. B. Reverso.

En la morfología microscópica, visualizada con un montaje con azul de lactofenol, se observan macroconidias equinuladas, fusiformes, de pared gruesa y de múltiples septos (Fig. 8). 


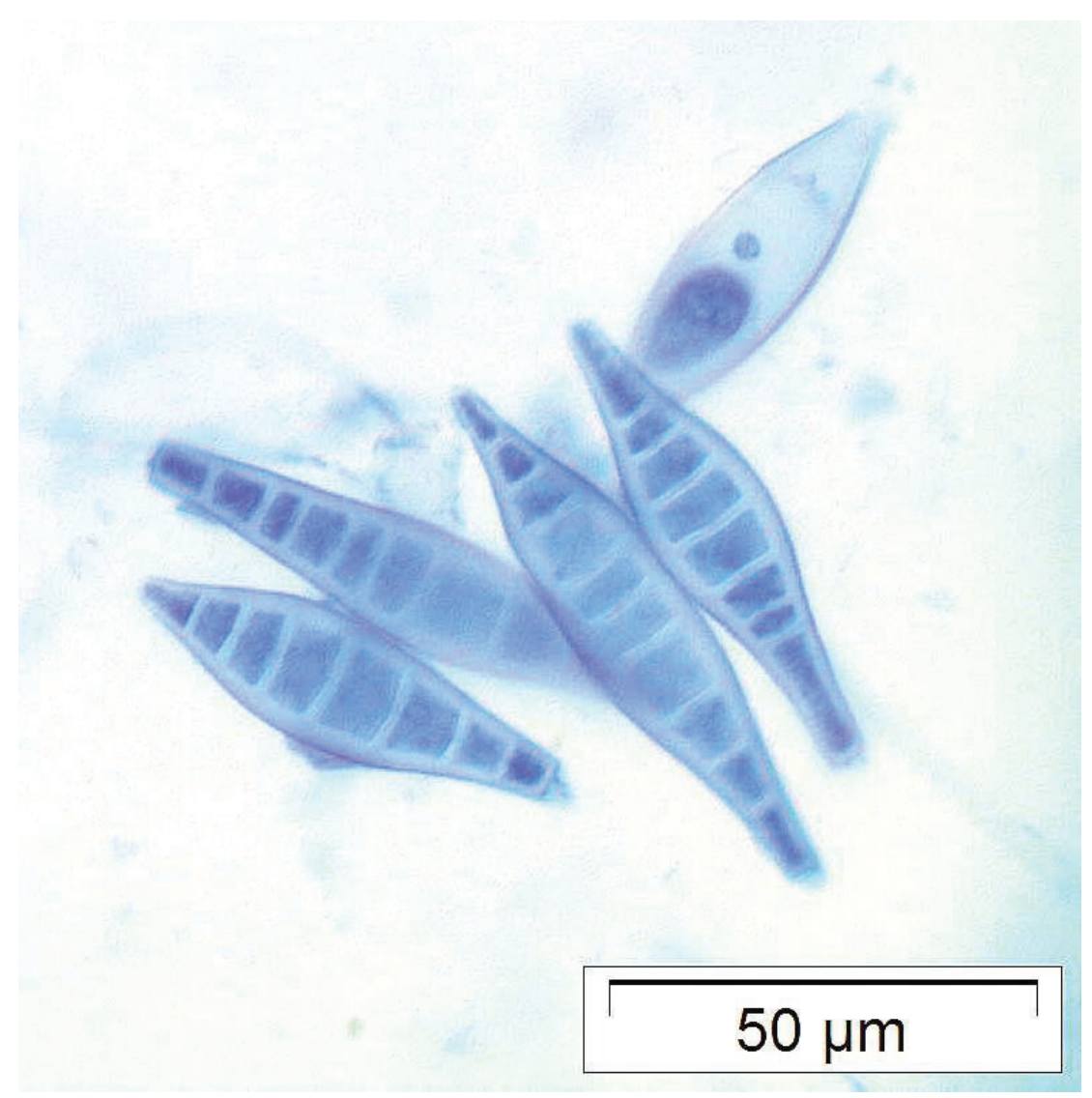

Fig. 8. Macroconidias fusiformes de Microsporum canis. Lactofenol azul, 400x.

Las características, tanto macroscópicas como microscópicas, de la colonia permitieron identificar al agente etiológico como Microsporum canis (de Hoog et al. 2014; Walsh et al. 2018a).

\section{Tratamiento y seguimiento clínico}

A partir del 25 de enero de 2019, la paciente fue medicada con itraconazol en suspensión oral (Itraskin, Drag Pharma, Lautaro, Chile); con una dosis de $15 \mathrm{mg} / \mathrm{kg}$ una vez al día y Trihepat ${ }^{\circ}$ (Labyes, Buenos Aires, Argentina) como protector hepático en suspensión oral $0.8 \mathrm{ml}$ dos veces al día. El 17 de febrero de 2019, la herida de la primera biopsia aún no había cicatrizado (Figura 9), la ALT empezaba a disminuir (199 U/L), pero el animal inició con anorexia y se volvió reacio al tratamiento, por lo cual, se cambió la presentación de los medicamentos a formulaciones magistrales de itraconazol en cápsulas de $50 \mathrm{mg}$, una vez al día, junto con comida grasosa durante dos meses; además, silimarina en cápsulas de 150 mg, una vez al día, durante un mes. Los propietarios no pudieron llevar la gata a control en abril; por consiguiente, estuvo sin protector hepático por un mes; en consecuencia, la medición de ALT se realizó hasta el 12 de mayo de 2019. Reveló un aumento (401 U/L); así mismo, el animal presentó poca mejoría clínica, incluso, perdió $0.5 \mathrm{~kg}$ de peso. 


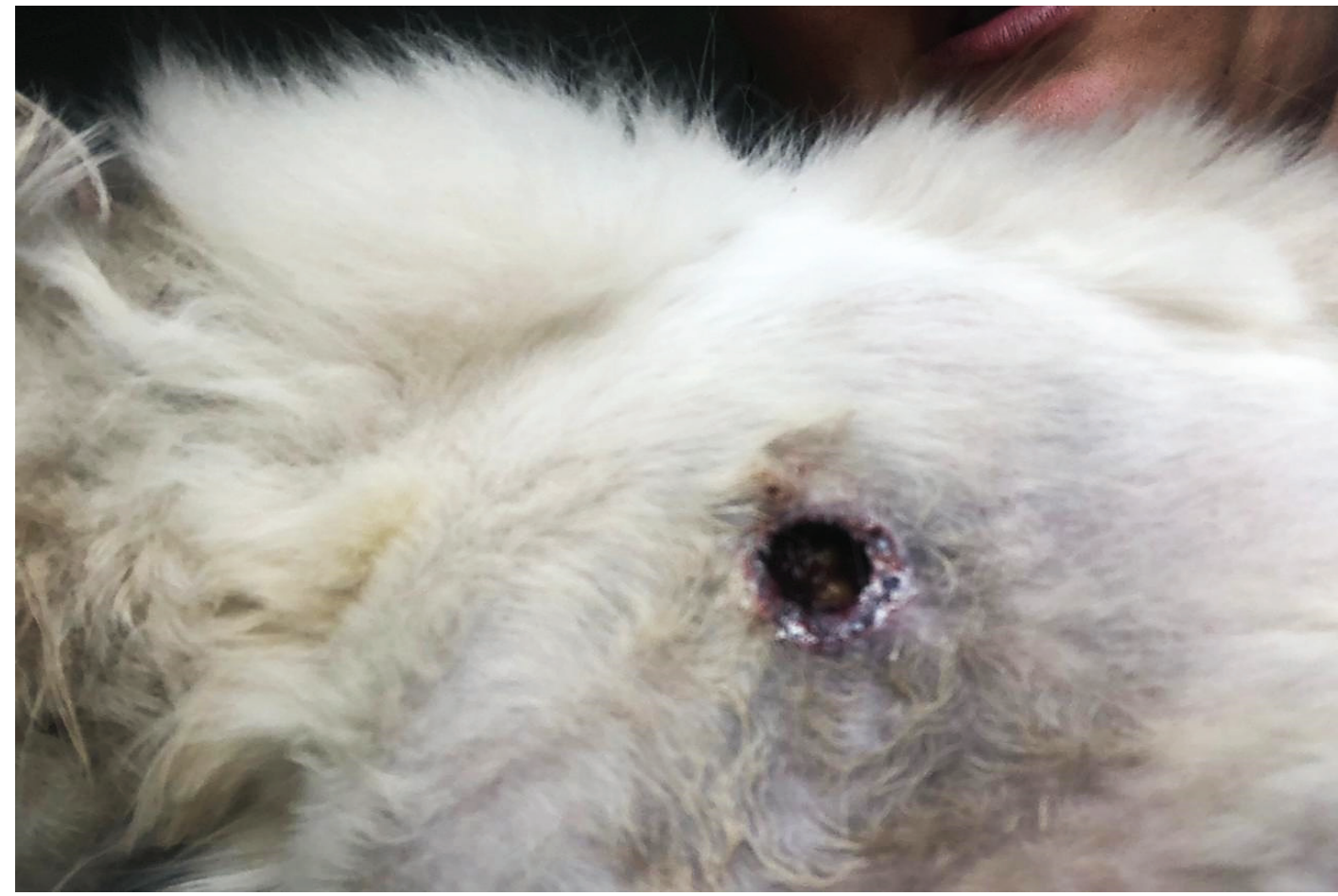

Fig. 9. Se observa la lesión producto de la debridación y lenta cicatrización del sitio donde se tomó la primera biopsia.

Debido al incremento de la ALT, la disminución de peso del animal y, al considerar que los pseudomicetomas se pueden presentar en órganos internos (Bianchi et al. 2017), se recomendó realizar un ultrasonido abdominal de control, el 25 de mayo de 2019. En este examen, no se presentan alteraciones significativas, solamente una masa libre, de origen desconocido, de ecogenicidad mixta, caudal al riñón derecho. Se decidió hacer una citología de esta masa, en lugar de una biopsia, dado que la primera es menos invasiva. No se había realizado pruebas de tiempos de protrombina y tromboplastina, porque la paciente había presentado dificultad para cicatrizar. Con la citología se confirmó que se trataba de un linfonodo con hiperplasia linfoide, probablemente asociada al proceso infeccioso que tenía el animal.

El 25 de mayo de 2019, debido a la poca mejoría clínica e incomodidad del animal al recibir el medicamento, los propietarios desistieron de continuar con la terapia antifúngica y el protector hepático. No se logró dar un seguimiento presencial posterior, debido a que la clínica veterinaria, a la cual acudían, cerró; sin embargo, el 7 de agosto de 2019, vía telefónica, los propietarios informaron que la paciente seguía con buen estado de ánimo y apetito, más la presencia de masas aún palpables. Posteriormente, el 29 de enero de 2020, la nueva clínica tratante informó, al Laboratorio de Micología EMV-UNA, que la paciente fue llevada a control médico porque el ánimo y apetito disminuyeron.

\section{Discusión}

La dermatofitosis es la micosis superficial más frecuente en mamíferos a nivel mundial; aunque la presentación clínica, en forma de pseudomicetoma dermatofítico, es poco común (Hubka et al. 2018). 
La gran mayoría de casos reportados ha sido en gatos persa (Bond et al. 2001; Pereira et al. 2006; Stanley et al. 2008; Chang et al. 2010; Nobre et al. 2010; Bernhardt et al. 2015; Bianchi et al. 2017; Duangkaew et al. 2017; Sousa Filho et al. 2017).

Dado que esta micosis inicia con la colonización del folículo piloso y que, en gatos de exteriores, se encuentra frecuentemente en el tórax dorsal, sugiere que la implantación de los microorganismos se da por medio de portadores asintomáticos, mediante rasguños por peleas o durante el apareamiento (Gross et al. 2005). En este caso, los propietarios reportaron que hacía, aproximadamente, dos años se había escapado de casa y regresó con varias lesiones, compatibles con arañazos; por lo cual, se cree esta fue la vía de entrada. Adicionalmente, los cultivos de uñas y cepillado de pelambre de los gatos resultaron negativos por dermatofitos y, aunque no se descarta que estos pudieran ser fuentes de infección en el pasado, al momento del muestreo, no representaban riesgo alguno de infección para los propietarios o para los gatos.

En la histopatología, se identificó una inflamación piogranulomatosa, característica de esta patología (Tostes \& Giuffrida 2003; Pereira et al. 2006; Kano et al. 2008) cuyo diagnóstico etiológico de pseudomicetoma dermatofítico por $M$. canis fue confirmado mediante el cultivo micológico; este hongo es el único agente involucrado en esta patología (Stanley et al. 2008; Chang et al. 2011; Bianchi et al. 2017).

El tratamiento, para este tipo de micosis, consiste en la administración de terapia antifúngica sistémica, junto con la remoción de los nódulos subcutáneos, siempre que sea posible, debido a la dificultad de resolver esta patología, exclusivamente con antimicóticos. Se recomienda remover las masas, incluyendo márgenes amplios de alrededor de $2 \mathrm{~cm}$ de piel, junto con fascia subyacente, e incluso músculo; esto para disminuir la posibilidad de recurrencia del pseudomicetoma (Chang et al.2010). Usualmente, estos tratamientos son largos; incluso tediosos para el animal y los propietarios; pueden llegar a afectar órganos como el hígado debido a lo prolongado de la terapia. En el caso del felino de Costa Rica, se eligió el itraconazol, por ser el de mayor éxito en la literatura consultada (Pereira et al. 2006; Kano et al. 2008; Chang et al. 2010; Sousa Filho et al. 2017), al tener buena biodisponibilidad en suspensión oral y en cápsulas, si se administra con comida grasosa (Plumb 2018a), también, por ser el de menor costo económico. Se consideró terbinafina por ser el de mejor efecto residual y menor toxicidad, pero al tener baja biodisponibilidad oral en gatos (Bond et al. 2001; Plumb 2018b; Seyedmousavi et al. 2018), la opción era inyectar diariamente; esto podría propiciar la propagación iatrogénica de pseudomicetomas en los sitios de inyección. No se realizó cirugía debido a los problemas para cicatrizar que presentó el animal; además, era posible que la piel no cediera suficiente para retirar la masa ubicada dorsal a la base de la cola, dejando muy justo los tejidos e influyendo en la movilización; por ende, el bienestar del animal y la cicatrización.

El pronóstico, en los casos de pseudomicetoma, es reservado, debido a lo complejo del tratamiento, cuya duración puede ser de entre seis hasta los 18 meses con antifúngico sistémico; además, en muchos de los casos, a pesar de la remoción quirúrgica y el tratamiento sistémico, ocurre recidiva (Pereira et al. 2006; Sousa Filho et al. 2017).

La gran mayoría de los casos reportados no ha tenido patologías concomitantes, con excepción de dos casos, en los cuales, el pseudomicetoma dermatofítico estuvo acompañado de blastomicosis; otro, en el cual, el gato tenía ácaros del género Notoedres (Duangkaew et al. 2017; Sousa Filho et al. 2017). En este caso, se descartaron otras enfermedades adicionales. 


\section{Conclusiones}

El diagnóstico de pseudomicetoma dermatofítico se debe realizar mediante histopatología y cultivo micológico, así como un seguimiento que incluya bioquímica sanguínea hepática y ultrasonido. Un manejo temprano de la enfermedad influye positivamente en el pronóstico, el cual es de carácter reservado debido a que el tratamiento involucra, tanto la remoción quirúrgica de los nódulos como la administración prolongada de antifúngicos, aun así la posibilidad de recidiva es alta. Se recomienda, a los clínicos de especies menores de Centroamérica, considerarla en el diagnóstico diferencial de nódulos cutáneos y abdominales de gatos, en especial los de raza persa.

\section{Conflicto de intereses}

Los autores declaran no tener conflicto de intereses.

\section{Agradecimientos}

A los propietarios de esta paciente por hacer el seguimiento de su mascota y permitirnos publicar la información. A la Dra. Rebeca Orias del Hospital Veterinario La Vete, por suministrar fotografías recientes del animal.

\section{Referencias}

Bernhardt, A., von Bomhard, W., Aintweiler, E., \& Tintelnot, K. 2015. Molecular identification of fungal pathogens in nodular skin lesions of cats. Med. Mycol. 53(2): 132-144. doi: 10.1093/mmy/myu082

Bianchi, M.V., Laisse, C.J.M., Vargas, T.P., Wouters, F., Boabaid, F.M., Pavarini S.P., Ferreiro, L. \& Driemeier, D. 2017. Intra-abdominal fungal pseudomycetoma in two cats. Rev. Iberoam. Micol. 32(2): 112-115. doi: 10.1016/j.riam.2016.10.001

Bond, R., Pocknell, A.M. \& Toze, C.E. 2001. Pseudomycetoma caused by Microsporum canis in a Persian cat: lack of response to oral terbinafine. J. Small Anim. Pract. 42(11): 557-560. doi: 10.1111/j.17485827.2001.tb06028.x

Calderón-Hernández, A, \& Urbina-Villalobos, A. 2018. Veterinary mycosis in a tropical country. 20th Congress of the International Society for Human and Animal Mycology. Amsterdam, The Netherlands. Abstract, Med. Mycol. 56: S1-S159. doi: doi.org/10.1093/mmy/myy036

Chang, S.C., Liao, J.W., Shyu, C.L., Hsu, W.L., \& Wong, M.L. 2010. Dermatophytic pseudomycetomas in four cats. Vet. Dermatol. 22(2): 181-187. doi: 10.1111/j.1365-3164.2010.00937.x

de Hoog, G.S., Guarro, J., Gené, J. \& Figueras, M.J. 2014. Microsporum canis. In: Atlas of clinical fungi: The ultimate bench tool for diagnostics, online version 4.0. Centraalbureau voor Schimmelcultures, Utrecht.

Duangkaew, L., Larsuprom, L., Kasondorkbua, C., Chen, C. \& Chindamporn, A. 2017. Cutaneous blastomycosis and dermatophytic pseudomycetoma in a Persian cat from Bangkok, Thailand. Med. Mycol. Case Rep. 15(1): 12-15. doi: 10.1016/j.mmcr.2017.01.001 
Goldberg, H. 1965. Brush technique in animals: finding contacts sources of fungus diseases. Arch. Dermatol. 92(1): 103.

Gross, T.L., Ihrke, P.J., Walder, E.J. \& Affolter, V.K. (Eds.). 2005. Skin Diseases of the Dog and Cat, 2nd Edition. Blackwell Publishing, Oxford. p 288-291.

Hubka, V., Peano, A., Cmokova, A. \& Guillot, J. 2018. Common and emerging dermatophytoses in animals: well-known and new threats. In: Seyedmousavi, S., de Hoog, G.S., Guillot, J., \& Vertweij, P.E (Eds.). Emerging and epizootic fungal infections in animals. Springer Nature, Cham. p 31-79. doi: $10.1007 / 978-3-319-72093-7 \quad 3$

Kano, R., Edamura, K., Yumikura, H., Maruyama, H., Asano, K., Tanaka, S. \& Hasegawa, A. 2008. Confirmed case of feline mycetoma due to Microsporum canis. Mycoses 52(1): 80-83. doi: 10.1111/j.1439-0507.2008.01518.x

Nobre, M., Mueller, E., Tillmann, M., Rosa, C., Guim, T., Vives, P., Fernandes, M., Madrid, I., Fernandes C. \& Meireles, M.C. 2010. Disease progression of dermatophytic pseudomycetoma in a Persian cat. Rev. Iberoam. Micol. 27(2): 98-100. doi: 10.1016/j.riam.2009.12.004

Pereira, A., Damico, C., de Souza, H., Corgozinho, K., Graça, R., Paes de Almeida, E. \& Ferreira, A.M. 2006. Pseudomicetoma dermatofítico causado por Microsporum canis em gato da raça persa. Acta Sci. Vet. 34(2): 193-196.

Plumb, D.C. 2018a. Itraconazole. In: Plumb’s Veterinary Drug Handbook, 9th Edition. Wiley-Blackwell, New Jersey. p. 642-645.

Plumb, D.C. 2018b. Terbinafine HCl. In: Plumb's Veterinary Drug Handbook, 9th Edition. WileyBlackwell, New Jersey. p. 1117-1119.

Seyedmousavi, S., Wiederhold, N.P., Ebel, F., Hedayati, M.T., Rafati, H. \& Verweij, P.E. 2018. Antifungal use in veterinary practice and emergence of resistance. In: Seyedmousavi, S., de Hoog, G.S., Guillot, J. \& Vertweij, P.E (Eds.). Emerging and epizootic fungal infections in animals. Springer Nature, Cham. p 359-402. doi: 10.1007/978-3-319-72093-7 16

Sousa Filho, R.P. de, Sampaio, K. de O., Alves, M.A.A., Nogueira, D.A.D., Viana, D. de A., de Cunha, M. G.M.C., \& da Silva, T.F.P. 2017. Pseudomicetoma dermatofítico disseminado em um gato persa - relato de caso. Arq. Ciênc. Vet. Zool. 20(1): 29-33. doi: 10.25110/arqvet.v20i1.2017.6317

Stanley, S.W., Fischetti, A.J. \& Jensen, H.E. 2008. Imaging diagnosis - sublumbar pseudomycetoma in a Persian cat. Vet. Radiol. Ultrasoun. 49(2): 176-178. doi: 10.1111/j.1740-8261.2008.00346.X

Tostes, R.A. \& Giuffrida, R. 2003. Pseudomicetoma dermatofítico em felinos. Ciênc. Rural. 33(2): 363-365.

Walsh, T.J., Hayden, R.T., \& Larone, D.H. 2018a. Microsporum canis. In: Larone's Medically Important Fungi: A guide to identification, 6th Edition. ASM Press, Washington D.C. p. 262.

Walsh, T.J., Hayden, R.T. \& Larone, D.H. 2018b. Stains. In: Larone’s Medically Important Fungi: A guide to identification, 6th Edition. ASM Press, Washington D.C. p. 27-28. 